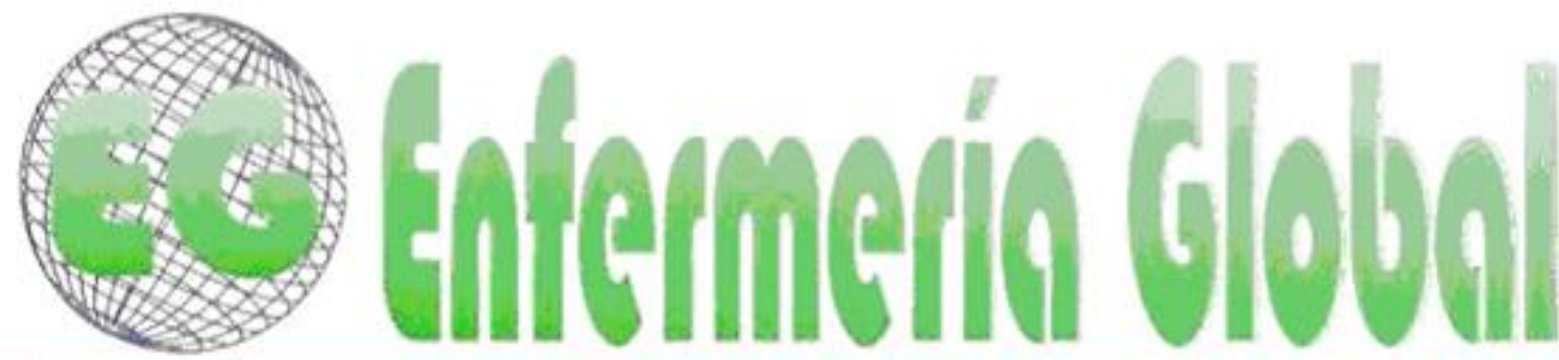

ISSN 1695-6141

Revista electrónica trimestral de Enfermeria

No34

Abril 2014

www.um.es/egloball

REVISIONES

\title{
Estado actual de la salutogénesis en España. Quince años de investigación
}

Current status of the salutogenesis in Spain. Fifteen years of research

\author{
${ }^{*}$ Ruano Casado, Luisa **Mercé Valls, Elisa \\ *Doctora. Profesora Titular del Departamento de Enfermería. Facultad de Enfermería y Podología. \\ Universitat de València. E-mail: luisa.ruano@uv.es **Enfermera. Residencia Asistida de $3^{\text {a }}$ Edad Lledó \\ de Castellón. Doctoranda Universitat de València. España.
}

Palabras clave: Salud Pública; Promoción de la salud; salutogénesis; sentido de coherencia; capacitación

Keywords: Public health; Health promotion; Salutogenesis; Sense of coherence; Training.

\section{RESUMEN}

Tras siglos de visión patogénica del cuidado de la salud, en los años 70 el sociólogo y médico Aaron Antonovsky introdujo un modelo de salud que con los años se ha convertido en un concepto establecido en la salud pública y la promoción de la salud, la 'Salutogénesis', que propone entender la salud como un continuo entre bienestar y enfermedad en lugar de una visión en que salud y enfermedad se excluyen mutuamente, coincidiendo con el actual enfoque de la Organización Mundial de la Salud.

Este trabajo tiene como objetivo principal realizar una revisión y análisis de su estudio y utilización en España, donde va introduciéndose de forma progresiva, con más de 10 artículos publicados, una conferencia europea, un simposio con expertos de todo el mundo, un seminario a nivel europeo, la creación de un grupo español de salutogénesis y con un convenio nacional de Universidades Saludables-Salutogénicas.

Es necesario trabajar desde las políticas públicas de salud, en coordinación con los servicios sanitarios y en la formación, remarcando el peso de las universidades, para capacitar a los futuros profesionales de enfermería desde la visión activa de promoción de la salud

\section{ABSTRACT}

After centuries with a pathogenic vision of care of health, in the 1970s the medical sociologist Aaron Antonovsky introduced a model of health that had become a concept established in the public health and the promotion of health, 'Salutogenesis', that proposes to understand health as a continuum between welfare and disease, instead of a vision where health and disease are mutually exclusive, by 
coinciding with the current approach of World Health Organization. The main goal of this paper is to lead a review and analysis of its study and use in Spain, where it is progressively introducing with more than 10 articles, an European Conference, a Symposium with experts from all over the world, an European Seminar, the creation of a Salutogenesis Spanish Group and with a National Convention of Healthy-Salutogenics Universities among other activities.

Is necessary to work from the public health policies, in coordination with the health services and education, highlighting the importance of the universities, in order to train future professionals from the active view of health promotion

\section{LA PROMOCIÓN DE LA SALUD Y EL NACIMIENTO DE LA SALUTOGÉNESIS}

Desde 1946, cuando se firmó la Carta Magna o Carta Constitucional de la Organización Mundial de la Salud, y se definió la salud como "El estado completo de bienestar físico, mental y social y no solamente la ausencia de afecciones o enfermedades" (1), se sucedieron hitos importantes en relación a la Promoción de la Salud como el informe Lalonde (1974), la primera Conferencia Internacional sobre Atención Primaria de Salud (1978), o la primera Conferencia Internacional de Promoción de la Salud (1986), donde se sintetizó la producción hasta ese momento y representó el consenso alcanzado por los representantes de 35 países presentes, identificando los campos de acción en la Carta de Ottawa: políticas públicas saludables, ambientes favorables a la salud, acción comunitaria, habilidades personales y reorientación del sistema de salud y recomendando la participación comunitaria en los procesos decisorios, en la planificación y en la implementación de las acciones de salud ${ }^{(2)}$

Paralelamente a todos estos acontecimientos, en los años 70, el médico y sociólogo Aaron Antonovsky, desarrolló su teoría de la salud, que denominó 'Salutogénesis' a partir de estudios que incluían a supervivientes del holocausto, en los que encontró que, tras haber sufrido los horrores de esta guerra, muchos de ellos no sólo se recuperaron y sobrevivieron, sino que prosperaron. Antonovsky propuso que deben existir factores que causan la salud ${ }^{(3,4)}$

En 1992, en un seminario que se celebró en la Oficina Regional de la OMS en Copenhague, fueron discutidas las teorías subyacentes de la investigación de promoción de la salud. El Dr. Antonovsky asistió a este taller y presentó su modelo como una dirección para la promoción de la salud ${ }^{(5)}$. Este modelo propone que el objetivo de la investigación en salud debe ser identificar, definir y describir las rutas, los factores y causas de la salud positiva para complementar nuestros conocimientos sobre cómo prevenir, tratar y gestionar la salud desde el punto de vida patogénico, centrado en el descubrimiento de las causas y los precursores de la enfermedad y la identificación de factores de riesgo de enfermedades ${ }^{(3,4)}$.

La teoría salutogénica no ha sido tan utilizada como se esperaba, a pesar de las similitudes teóricas con la esencia de la Carta de Ottawa, ${ }^{(6)}$ debido en parte a que el enfoque de salud que ha rodeado durante muchos años tiende a valorar y estudiar todo aquello que nos rodea y que se considera que puede dañarnos o hacernos enfermar, pero no aquello que puede ser saludable. La definición negativa de salud como ausencia de enfermedad es poco operativa, ya que nos obliga a diferenciar entre lo "normal" y lo "patológico" (diferencia que no siempre es posible y que está afectada por el concepto de normalidad variable) ${ }^{(7)}$.

En las últimas décadas este modelo ha recibido una mayor atención y se está utilizando en diferentes ámbitos como es la prevención, la salud pública, la neurología 
y la psicoterapia entre otros, con el enfoque en el ser humano y los derechos humanos. El individuo se convierte en un sujeto activo y participante. La tarea de los profesionales es apoyar y ofrecer opciones, señalando los principales factores determinantes de la salud, para que la gente tome conciencia de la ellos y pueda utilizarlos ${ }^{(8,9)}$.

En 2007, el Grupo Temático de Trabajo sobre Salutogénesis se estableció en la International Union for Health Promotion and Education (IUHPES), 19a Conferencia Mundial de Promoción de la Salud y de la Educación, en Canadá. El primer encuentro internacional de investigación y seminario internacional de investigación se llevó a cabo en 2008 en Helsinki, Finlandia. Desde ese momento se han realizado diversos seminarios y congresos ${ }^{(10)}$.

A pesar de haber sido publicados más de 500 trabajos relacionados con el modelo, diversos seminarios y conferencias en el mundo, en nuestro país, la producción científica y las políticas llevadas a cabo parecen ser escasas, razón por la cual, el objetivo principal de este trabajo es realizar una revisión y análisis del estudio de la salutogénesis y su utilización en España.

\section{METODOLOGÍA}

Se consultaron las bases de datos de MEDLINE, CINAHL (Cumulative Index to Nursing and Allied Health Literatura), Psycinfo, La Biblioteca Cochrane Plus, BVS (Biblioteca Virtual en Salud), Teseo y bases de datos del CSIC (Consejo Superior de Investigaciones Científicas), portales de difusión científica como Dialnet y bibliotecas de diversas Universidades españolas.

La estrategia de búsqueda para las bases en inglés, MEDLINE y CINAHL se elaboró con los descriptores Medical Subject Heading (MESH) «Spain», «Sense of coherence» conjugando los descriptores con el operador AND. En el resto de bases de datos en lengua inglesa se utilizaron los mismos descriptores. Se incluyeron todos los artículos sin restricción respecto a las fechas de publicación para todas las bases de datos internacionales. En relación a las bases en lengua española, en BVS los Términos Descriptores en Ciencias de la Salud (DeCS) fueron «sentido de coherencia», «promoción de la salud» y «salud pública» y en el resto se utilizaron los términos libres «España», "salutogénesis», "Anstonovsky», "sentido de coherencia» y «orientación salutogénica», realizando múltiples combinaciones.

\section{RESULTADOS}

\subsection{Artículos y trabajos sobre salutogénesis realizados en España}

En 1997, se presentó un trabajo en el que se abordaba la relación entre salud, el sentido de coherencia, la personalidad resistente y la autoestima, encontrándose numerosas correlaciones entre ellos y sus componentes respectivos. Se tradujo y adaptó al español el cuestionario de medida de Sentido de Coherencia (SOC) en su versión de 29 ítems de Antonovsky ${ }^{(4)}$, el cuestionario reunía los requisitos de consistencia interna en sus tres componentes. Se informó, por primera vez en España de las características psicométricas del cuestionario ${ }^{(11)}$.

En 1999, se estudió el papel del SOC como mediador entre el estrés y el síndrome de burnout en el trabajo y la salud percibida, con 115 profesores de educación primaria, 
en el que utilizaron la misma versión del cuestionario. Se mostró que el SOC actuaba, dependiendo del nivel en que se presentase, como inhibidor o facilitador del estrés de rol, de burnout y de la sintomatología percibida, especialmente al considerar específicamente las dimensiones del constructo ${ }^{(12)}$.

Posteriormente, en 2006, se examinaron las relaciones establecidas entre las variables que intervienen en el proceso de adaptación familiar al estrés que supone el cuidado de un hijo con autismo, con una muestra de 39 madres. Se utilizó el cuestionario de medida del SOC de 29 ítems. Se observó que los apoyos y el SOC tenían una relación directa y negativa con el estrés, actuando como variables moduladoras. Sin embargo, la relación directa y negativa entre estresor y SOC les sugería que a medida que aumentaba la gravedad del trastorno y los problemas de conducta, se observaba una disminución en los niveles de SOC. Respecto a la relación entre SOC y apoyos, existía una relación directa teórica que no resultó significativa en el contraste empírico ${ }^{(13)}$

En 2007, se estudiaron la aceptabilidad, consistencia interna, asunciones escalares, estructura factorial, validez de constructo y precisión de la escala de SOC de Antonovsky, de 13 ítems (OLQ-13) en 419 adultos mayores (70 años) de España. Se ofrecieron datos óptimos en fiabilidad y nuevos aportes de validez convergente del SOC, con medidas autorreferidas de salud física, calidad de vida y depresión, concluyendo que el OLQ-13 es una escala válida y fiable para su uso en poblaciones ancianas españolas, incluidos grupos con bajo nivel de instrucción ${ }^{(14)}$

En este mismo año, en 2007, se realizó otro estudio con alumnos de la Escuela Universitaria de Ciencias de la Salud del Campus de Ponferrada de la Universidad de León, con los objetivos de comprobar la fiabilidad del cuestionario SOC en alumnos universitarios de Ciencias de la Salud y hallar la relación del sentido de coherencia con su salud psicológica. La muestra fue de 261 alumnos. Se utilizo el cuestionario SOC-13. Los valores de Sentido de Coherencia encontrados se correspondían con niveles altos y se observó una relación estadísticamente significativa entre el sentido de coherencia y la salud psicológica ${ }^{(15)}$.

En 2008, en la Universidad de Oviedo se abordó la relación entre la salud, por un lado, y las dimensiones de personalidad y el sentido de coherencia, por otro, en pacientes diagnosticados de fibromialgia. En este caso, los autores refieren utilizar el SOC-29, Los resultados mostraron un sentido de coherencia inestable en las pacientes con fibromialgia $=60)$ respecto a las pacientes con artritis reumatoide $(n=14)$ y los controles sanos $(n=13)$. Las pacientes fibromiálgicas destacaban por la menor capacidad para percibir los problemas como inteligibles (comprensibilidad), que los recursos para controlarlos estaban disponibles (manejabilidad) y que merecía la pena afrontarlos (significado) y se afirmaba, tras los resultados obtenidos, que las pacientes con fibromialgia presentaban rasgos de personalidad y creencias de control que podrían actuar de forma patogénica favoreciendo la aparición de la enfermedad y alterando la percepción subjetiva de la misma ${ }^{(16)}$.

En 2011, se presentó un estudio cuyo objetivo fundamental era determinar la relación entre actividad física, salud y sentido de coherencia en una muestra de 80 adultos mayores de 60 años que se percibían como sanos y que asistían diariamente a una estancia diurna especial para personas mayores para realizar diversos tipos de actividades. Se aplicaron dos cuestionarios, siendo uno de ellos el cuestionario SOC. Los resultados indicaron que la generalidad de los entrevistados, aun cuando 
padeciesen alguna enfermedad crónica degenerativa, se autopercibían como sanos. Las personas de la muestra que realizaban alguna actividad física, mostraban, de manera estadísticamente significativa mayor sentido de coherencia que aquellas que no lo hacían ${ }^{(17)}$.

Ese mismo año, se investigó la relación del síndrome de burnout con la personalidad resistente y el sentido de coherencia, con el fin de determinar si éstas podían ser entendidas como predictores del burnout. Se realizó un estudio descriptivo y transversal. 144 profesionales sanitarios de Cuidados Intensivos que respondieron a la adaptación española del Cuestionario de Orientación a la vida (OLQ-13) ${ }^{(13)}$. Se detectó un grado medio-alto de burnout entre los participantes y se observó una correlación negativa entre la personalidad resistente y burnout. En relación al SOC, se mostró que la personalidad resistente y la comprensibilidad, componente del sentido de coherencia, modulaban los efectos de los estresores laborales en el síndrome de burnout ${ }^{(18)}$.

En 2012, se publicó un estudio sobre la influencia de varias dimensiones de familias en el sentido de coherencia (SOC) en la adolescencia, controlando los posibles efectos de las variables demográficas, el género y la edad. Se presentó otro estudio, con la misma población, que estudiaba la influencia del vecindario en el desarrollo del SOC. La muestra final, en ambos, fue de 7580 adolescentes con edades entre 13 y 18 años y se utilizó el cuestionario SOC-29. En el primer estudio, los resultados mostraron que no existían diferencias importantes de género en los niveles de la SOC, pero sí por edad. También sugerían que el contexto familiar juega un papel importante en proporcionar experiencias significativas para el desarrollo de un fuerte SOC en la adolescencia. En el segundo estudio se mostraba la importancia de relaciones sociales, un barrio seguro y la existencia de lugares adecuados (de recreo) para el encuentro con sus vecinos implicaban beneficios con el $\operatorname{SOC}^{(19,20)}$.

\subsection{Revisiones}

En 2009, se revisaron cinco artículos, aunque ninguno español, sobre el conocimiento del papel que desempeñaba el sentido de coherencia en el ámbito laboral. A pesar de que los resultados de los estudios anteriores indicaban su relación, concluyeron que la investigación disponible no es del todo convincente, siendo necesarios estudios longitudinales ${ }^{(21)}$.

En 2010, se publicó una revisión en la que se relacionaban el modelo de activos, la salud positiva, la resilencia y la salutogénesis, sobre todo en la infancia y adolescencia, presentando como factible generar un mapa de activos sustentados en las potencialidades y los intereses de la comunidad como alternativa a los tradicionales diagnósticos de salud basados en el déficit. Se mostraron las diferencias entre las teorías y la práctica del modelo del déficit y el basado en la salud ${ }^{(22)}$.

En 2011, se publicó una revisión sobre los trabajos publicados en España con utilización del cuestionario SOC, donde se estudió el estado del modelo en el país, su evolución a lo largo de la historia y su relación con otras teorías de promoción de la salud, desglosándose los componentes del modelo y la relación de este enfoque con la vida, la salud, la calidad de vida y sus implicaciones y retos en la salud pública ${ }^{(23)}$

En 2012, en una revisión que incluía 5 estudios descriptivos y una revisión sistemática de 15 estudios, ninguno de ellos realizado en España, se presentó la relación entre 
salutogénesis y el colectivo enfermero con o sin trabajo. El SOC se relacionó con la salud mental, el bienestar y la calidad de vida y se mostraba como factor protector frente a los factores estresantes provenientes del ambiente laboral y de las dificultades de conciliación laboral y familiar con niveles altos y se asociaba con problemas de salud como el burnout o la depresión y con determinadas características de la personalidad con niveles bajos ${ }^{(24)}$.

\subsection{Posters}

En el IV Seminario de Investigación en Salutogénesis (2011), se presentó un poster cuyo objetivo se centraba en un análisis multidimensional de la calidad de vida familiar (FQL), basado en un modelo que propone que la adaptación depende de las interrelaciones entre las características del niño, el apoyo social, la percepción del problema (sentido de la coherencia) y las estrategias de afrontamiento. La muestra fue de 59 padres y 59 madres. Los datos empíricos se ajustaban al modelo teórico y había diferencias de género. El SOC presentaba un papel fundamental en la adaptación como un moderador de los efectos que los problemas de conducta del niño producen en la calidad de vida familiar ${ }^{(25)}$.

En el mismo Seminario, otro Poster presentaba un Plan de Salud, siguiendo los principios de Salutogénesis, elaborado por el Consejo de Salud del Gobierno Regional de Andalucía. Se mostraba a la Escuela Andaluza de Salud Pública (EASP) como una de las instituciones líderes en España en la gestión de conocimientos del modelo salutogénico, desarrollando la investigación de los conocimientos existentes sobre Salutogénesis y el establecimiento de estrategias de formación de profesionales sanitarios $^{(26)}$.

\subsection{Tesis y trabajos fin de Máster}

En la Universidad Politécnica de Madrid, en 2009, se presentó una Tesis Doctoral Europea. Se desarrolló y evaluó un programa basado en principios y métodos de la acción psicosocial y de la terapia a través del deporte y del movimiento, juegos y deportes, técnicas participativas y técnicas grupales, con 56 mujeres indígenas de dos grupos de la zona rural, en contextos de violencia y de conflicto en el Departamento de Sololá en Guatemala, C.A. Con una muestra de 508 personas, se utilizaron las versiones SOC-29 en la muestra de referencia $(n=452)$ y SOC-13 pre y post intervención $(n=33)$. En el análisis de los resultados del programa se mostró una mejora a nivel personal tanto en recursos cognitivos como psicológicos. La autoestima y el Sentido de Coherencia (especialmente la 'comprensibilidad') aumentaron de forma significativa y se encontraron diferencias entre los dos grupos de intervención $^{(27)}$.

En 2009, en otra Tesis Doctoral, en la Universidad de León, se estudiaron las relaciones entre la salud psicológica, estrés, afrontamiento, salud psicológica, sentido de coherencia, dimensiones de personalidad y autoestima, el cansancio emocional y el afrontamiento en 960 estudiantes universitarios. Para medir el Sentido de Coherencia se utilizó una versión distinta a la de Virués-Ortega y col. ${ }^{(19)}$ del SOC-13, con buenas propiedades psicométricas y una confirmación de la estructura de tres dimensiones. Mostraron que existía una relación estadísticamente significativa con las estrategias de afrontamiento, el nivel de estrés, el nivel de afrontamiento, estabilidad emocional, el neuroticismo, la extraversión y psicoticismo ${ }^{(28)}$. 
En 2010, en un Trabajo Fin de Master en la Universidad de Gerona, se estudió el SOC en 55 profesionales de enfermería de los cuatro Centros de Atención Primaria (CAP) de la ciudad. Se utilizó el cuestionario SOC-13 ${ }^{(19)}$. El SOC resultó moderadoalto, el más alto en estudios similares, pero no se relacionó estadísticamente con el resto de variables ${ }^{(29)}$.

\subsection{Seminarios, conferencias y congresos}

En Granada, el 27 y 28 de enero de 2010 se realizó un seminario dirigido a expertos en Prevención de la Salud. Se analizó la relación de los jóvenes y la salud y el enfoque de la salutogénesis en la infancia y la adolescencia, y la relación entre variables de salud personales, familiares, escolares y sociales, a la luz de los resultados del Estudio HBSC (Health Behaviour in School-aged Children), sobre conductas de los escolares relacionadas con la salud. El Dr. Lindström, que habló de la dificultad de los Facultativos para cambiar la visión y unir la vida con la salud, y no con las enfermedades y la muerte ${ }^{(30)}$.

En abril de 2010, en Sevilla, se realizó el symposium 'Health Assets for Young People's Wellbeing', con el objetivo general de contribuir a la promoción de los enfoques basados en activos para el bienestar de los jóvenes, destacando las acciones requeridas por investigadores, responsables políticos y profesionales para convertirse en una realidad ${ }^{(31)}$.

El 28 y 29 de junio de 2010, tuvo lugar en Madrid la conferencia 'Mental Health and Well-being in Older People -Making it Happen'. Se concluyó que el cambio demográfico de la Unión Europea se enfrenta a que en las próximas décadas habrá un mayor número de trabajadores de más edad y jubilados y serán necesarias mejores políticas para asegurar una buena salud mental y el bienestar de esta población, promocionándose las asociaciones activas, redes de colaboración e intercambio. Se propuso aplicar los principios salutogénicos dentro de la práctica profesional, incluyendo a las propias personas mayores en el desarrollo de la política y la práctica ${ }^{(32)}$

\subsection{Promoción desde las políticas de salud}

En 2011, siguiendo las teorías de la salutogénesis y el modelo de activos en salud, el Ministerio del Interior creó la Guía para la Promoción de la Salud Mental en el Medio Penitenciario. Se propone que las personas se adapten a los retos de la vida en prisión y adquieran el sentido de la coherencia a través de la comprensión, manejabilidad y significación: que lo que hagan sea satisfactorio y valioso, generando salud a través de las relaciones y del espacio vital penitenciario, modificando el entorno, reorientando los servicios y la atención en salud hacia el bienestar, a pesar de los muchos "pesares-obstáculos" con los que puedan tropezar en un centro penitenciario ${ }^{(33)}$.

El 10 de octubre de 2011, se firmó el Acuerdo Marco para el Desarrollo de la Red Española de Universidades Saludables (REUS), considerándolas como marco idóneo de actividad laboral, social y cultural, viendo la salud desde una orientación salutogénica, implicando no sólo sentar los medios que conduzcan a una educación para la salud y la promoción de la salud de sus trabajadores y estudiantes, sino también integrar la salud y el bienestar en la cultura universitaria, en los procesos y en 
el sistema universitario global ${ }^{\left({ }^{34}\right)}$. Paralelelamente, durante los últimos años, se han puesto en marche la Red Andaluza (RAUS), la Red Catalana (RED CAT) y la Red Madrileña de universidades saludables (REMUS).

En Julio de 2012, se celebró en Gerona la reunión constitutiva del Grupo de Trabajo en Salutogénesis, impulsado por la Cátedra de Promoción de la Salud y vinculado con el Grupo de Trabajo Global de la Unión Internacional de Promoción de la Salud y Educación para la Salud (UIPES). El objetivo fundamental fue compartir experiencias, publicaciones y fondo documental con grupos de trabajo en Salutogénesis del estado español y Latinoamérica, coordinados por el Equipo de Investigación en Promoción de la Salud, del Centro de Investigación Folkhälsan (Finlandia) ${ }^{(35)}$.

En 2012, en la declaración de Girona, se afirmó que para reformular la forma de entender y promover la salud, debemos orientar la mirada hacia el enfoque salutogénico y los activos en salud. Una estrategia global, intersectorial y de acción local y una invitación a la participación de los diferentes actores de la ciudadanía que pueden movilizarse desde escenarios saludables, incluido el entorno digital, desarrollando siempre políticas saludables donde la opción más saludable sea siempre la más fácil de tomar, situando en el centro de las políticas al ser humano como ciudadano consciente ${ }^{(36)}$.

\subsection{Programas y actividades}

Desde 2009, en Asturias, se está elaborando el mapa de activos en salud de la provincia. Se inició en las Jornadas de Participación y Actividades Comunitarias en Salud que se desarrollaron en 2009, iniciando un proceso para la recogida de información de las diferentes actividades comunitarias en salud de las que se dispone, estableciendo al final una base de datos (denominada Asturias Actúa, integrada dentro del Observatorio de Salud en Asturias) que cuenta con 101 actividades. Se han iniciado, conjuntamente con Partycipa, diferentes mapeos comunitarios en algunos puntos de Asturias ${ }^{(37)}$.

\section{CONCLUSIONES}

En España, la producción científica respecto al modelo salutogénico aunque en un estado incipiente, ha dado pasos relevantes, como la adaptación del cuestionario SOC en población española en sus dos versiones. Los estudios realizados hasta el momento, con muestras de población variadas, han sido enfocados en contextos laborales, familiares, universitarios y sociales y las políticas de salud, al igual que los programas y actividades que en este momento se están ejecutando, son aún tímidas, pero con un futuro esperanzador.

Es necesario el desarrollo sistemático de indicadores salutogénicos en el campo de la salud pública y el fortalecimiento de la perspectiva de promoción de la salud en el futuro, para desarrollar, educar y capacitar al personal y a los pacientes y alentar a los grupos académicos y profesionales a investigar y escribir acerca de prácticas salutogénicas eficaces, tal y como se destacó en el Modelo de Desarrollo de la Salud en Europa (EUHPID) ${ }^{(38)}$. Por otro lado, tal y como afirmo el Dr. Lindström, una política de salud con enfoque salutogénico conseguiría beneficios económicos, ya que con una buena salud mental y una buena calidad de vida gracias a un buen nivel de SOC, las personas enfermarían en menos ocasiones y habría menos incapacidades o prejubilaciones por enfermedad, siendo necesario para ello la capacitación de los 
profesionales, para paliar las dificultades que tienen los Facultativos, propiciando el cambio hacia una concepción de unir la vida con la salud, y no con las enfermedades y la muerte ${ }^{(30)}$.

El primer pilar en el que asentar esta nueva visión de promoción de la salud debería ser la educación, capacitando y motivando a los estudiantes de ciencias sociales y de la salud en general, y de enfermería en particular, a utilizar un pensamiento crítico y reflexivo, con una participación activa en su aprendizaje.

\section{REFERENCIAS BIBLIOGRÁFICAS}

1. World Health Organization. Preamble To the Constitution of the World Health Organization as adopted by the Intersectorial Health Conference, New York, 19-22 June, 1946: signed on 22 July 1946 by the representatives of 61 States (Oficial Records of the World Health Organization, no. 2, p.100) and entered into force on 7 April 1948.

2. Health Organization. Ottawa charter for health promotion: an International Conference on Health Promotion, the move towards a new public health, November 17-21 1986. Geneva: World Health Organization, 1986.

3. Antonovsky A. Health, Stress and Coping. San Francisco: Jossey-Bass; 1979.

4. Antonovsky A. Unraveling the Mystery of Health. How People Manage Stress and Stay Well. San Francisco: Jossey-Bass; 1987.

5. Antonovsky, A. The salutogenic model as a theory to guide health promotion. Health Promotion International. 1996; 11, 11-18.

6. Eriksson M, Lindstrom B. A salutogenic interpretation on the Ottawa Charter. Health Promot Int. 2008; 23: 190-98.

7. Moreno B, Garrosa E y Gálvez M. Personalidad positiva y salud. En: Florez-Alarcon L, Mercedes Botero M y Moreno Jimenez B. 2005: Psicología de la salud. Temas actuales de investigación en Latinoamérica. 2005; p59-76.

8. Eriksson, M. and Lindström, B. Antonovsky's Sense of Coherence Scale and the relation with health-a systematic review. Journal of Epidemiology and Community Health. 2006; 60, 376-381.

9. Lindström B, Eriksson M. Guía del autoestopista salutogénico. Camino salutogénico hacia la promoción de la salud. Documentia Universitaria. Girona. Universitat de Girona. 2011. ISBN: 978-84-9984-058-1.

10. International Union for Health Promotion and Education (IUHPE). Resolutions presented at the IUHPE General Assembly. Promotion \& Education. Vol. XIV, NO. 3. 2007

11. Moreno-Jiménez $B$, Alonso $M$, Álvarez. Sentido de la coherencia, personalidad resistente, autoestima y salud. Rev Psicol Salud.1997; 9: 115-37.

12. Moreno-Jiménez B, González JL, Garrosa E. Burnout docente, sentido de la coherencia y salud percibida. Rev Psicopatol Psicol Clín. 1999; 4: 163-80.

13. Pozo P, Sarriá E y Méndez L. Estrés en madres de personas con trastornos del espectro autista. Psicothema. Vol. 18, no 3, pp. 342-347; 2006. [Citado el 02 de febrero de 2012]. Disponible en: http://www.psicothema.com/pdf/3220.pdf.

14. Virues-Ortega J, Martinez-Martin P, Del Barrio JI, Lozano Lm. Validación transcultural de la Escala de Sentido de Coherencia de Antonovsky (OLQ-13) en ancianos mayores de 70 anos. Med Clin (Barc). 2007; 128: 486-92.

15. Fernández M E y cols. Sentido De Coherencia Y Salud Percibida en Alumnos Universitarios De Ciencias De La Salud. Asociación Nacional de Enfermería de Salud 
Mental [en línea]. 2007. [Citado el 14 de agosto de 2012]. Disponible en: http://www.anesm.net/anesm/contents/html.php?archivo= congresos premiosalaman ca1.

16. Besteiro, J Álvarez M, Lemos S, Muñiz J, Costas C, Weruaga A. Dimensiones de personalidad, sentido de coherencia y salud percibida en pacientes con un síndrome fibromiálgico International Journal of Clinical and Health Psychology, Vol. 8, Núm. 2, mayo-sin mes, 2008, pp. 411-427. ISSN 1697-2600

17. Hernández Z E. Actividad física, salud y sentido de coherencia y salud en personas mayores. En: XIII Congreso Andaluz de Psicología de la Actividad Física y el Deporte. Sevilla España, 17-19 de Noviembre de 2011. ISBN: 978-84-939424-1-0

18. García R M, Robles H. Personalidad resistente y sentido de coherencia como predictores de burnout en personal sanitario de cuidados intensivos. Ansiedad y estrés. ISSN 1134-7937. 2011; 17, № 2-3, págs. 99-112.

19. García-Moya I, Rivera F, Moreno C, Lindström B, Jiménez-Iglesias A. Analysis of the importance of family in the development of sense of coherence during adolescence. (Abstract). Scand J Public Health. 2012 Jun;40(4):333-9. doi: $10.1177 / 1403494812449924$.

20. García-Moya, I., Braun-Lewensohn, O. y Moreno, C. (2012). What's the role of neighborhood risks and assets in the development of SOC during adolescence? Health Promotion Research-An International Forum in conjuction with the 5th International Research Seminar on Salutogenesis. Trondheim, 6-9 Agosto de 2012.

21. Rodríguez-Blázquez C, Virués-Ortega J. Factores conductuales y estado de salud: posibilidades en prevención del constructo sentido de coherencia. [Artículo de opinión] Archivos de prevención de riesgos laborales, ISSN 1138-9672, Vol. 12, №. 2, 2009, págs. 88-90. [Citado el 20/01/2012]. Disponible en: http://www.scsmt.cat/pdf/352.pdf.

22. Hernán M, Lineros C. Los activos para la salud. Promoción de la salud en contextos personales, familiares y sociales. Rev Fundesfam. 2010; 2. [Citado el 30 de agosto de 2012]. Disponible en:

www.fundesfam.org/REVISTA\%20FUNDESFAM\%201/007revisiones.htm.

23. Rivera $F$, Ramos $P$, Moreno $C$ y Hernan $M$. Análisis del modelo salutogénico en España: aplicación en salud pública e implicaciones para el modelo de activos en salud. Rev. Esp. Salud Publica [online]. 2011, vol.85, n.2, pp. 129-139 . [Citado el 10 de septiembre de 2012]. Disponible en:

<http://scielo.isciii.es/scielo.php?script=sci_arttext\&pid=S1135-

$57272011000200002 \&$ Ing=es\&nrm=iso $>$. ISSN 1135-5727.

24. Malagón-Aguilera M C, et al. El sentido de coherencia en el colectivo enfermero. Enferm Clin. 2012. [Citado el 01 de Julio de 2012]. Disponible en:

http://dx.doi.org/10.1016/j.enfcli.2012.06.002.

25. Pozo P y Sarriá E. Quality of Life and Gender differences in Parents of Children with Autism Spectrum Disorders (ASD) in Spain: the Moderator role of Sense of Coherence. En: 4th International Research Seminar on Salutogenesis [Poster presentation]. 2011.

26. Hernán M, Mena A L. Starting an strategy to Implement the Salutugenic Model in Andalusia. En: 4th International Research Seminar on Salutogenesis [Poster presentation]. 2011.

27. Ley C. Acción psicosocial a través del movimiento, juego y deporte en contextos de violencia y de conflicto. Investigación sobre la adecuación sociocultural de la 'terapia a través del deporte' y evaluación de un programa con mujeres en Guatemala. [Tesis doctoral europea]. Universidad Politécnica de Madrid. 2009.

28. Fernández Martínez M E. Estrés percibido, estrategias de afrontamiento y sentido de coherencia en estudiantes de enfermería: su asociación con salud psicológica y estabilidad emocional. [Tesis Doctoral]. Universidad de León. 2009. 
29. Malagón M C. SOC a la professió infermera. [Trabajo Fin de Master]. Universitat de Girona. 2010. [Citado el 02 de julio de2012]. Disponible en:

http://dugidoc.udg.edu/bitstream/handle/10256/3210/Carme_Malagon_PROFESSIO_I NFERMERA..pdf?sequence $=1$.

30. Lindström B. Enfoque de la salutogénesis en la infancia y la adolescencia. El río de la vida y la promoción de la salud en todas las políticas. En: Seminario avanzado sobre promoción de salud, activos para la salud y salutogénesis. Experiencias en Andalucía y Europa. [online] Granada, 27 y 28 de enero de 2011.

31. Health Assets for Young People's Wellbeing. Symposium. Seville, Spain, 28-30 April 2010.

32. Billings J, Hashem F. Literature Review - Salutogenesis and the Promotion of Positive Mental Health in Older People. EU Thematic conference "Mental Health and Well-being in Older People - Making it Happen". Madrid: European Commission Directorate-General for Health and Consumers and the Spanish Ministry of Health and Social Affairs; 2009 [Citado 12 de agosto 2012]. Disponible en:

http://ec.europa.eu/health/mental_health/docs/older_salutogenesis.pdf

33. Equip Vincles Salut. Guía para la promoción de la salud mental en el medio penitenciario. Madrid: Ministerio del Interior. Secretaria General de Instituciones Penitenciarias. 2011; 198 p.

34. Acuerdo Marco de Colaboración entre el Ministerio de Sanidad, Política Social e Igualdad, el Ministerio de Educación y la Conferencia de Rectores de las Universidades Españolas para el Desarrollo de la Red Española de Universidades Saludables. Madrid, 10 de octubre de 2011. [Citado el 02 de marzo de 2012]. Disponible en:

http://www.educacion.gob.es/dctm/ministerio/educacion/universidades/educacionsuperior-universitaria/universidades-

saludables/convenio.pdf?documentld=0901e72b81238070

35. Creen a Girona el primer grup de I'Estat que buscarà estratègies per millorar la salut. La Càtedra de Promoció de la Salut de la UdG promourà la salutogènesi a través d'aquest equip. Diari De Girona. [En prensa] 03/09/2012.

36. Declaración de Girona. En: I Simposio de Promoción de la Salud en el Arco Mediterráneo. 30 de Mayo 2012. [Citado el 17 de septiembre de 2012.]. Disponible en: http://www.udg.edu/LinkClick.aspx?fileticket=e5AkwLUI0Qc\%3D\&tabid=18785\& language $=$ es-ES.

37. Asturias Actúa. En: Observatorio de Salud en Asturias. Gobierno del Principado de Asturias. [en línea] 2009.

38. Bauer G, Davies Jk, Pelikan J, on behalf of the EUHPID Theory Working Group and The EUHPID Consortium.The EUHPID health development model for the classification of public health indicators. Health Promot Int. 2006; 21:153-159.

ISSN 1695-6141

(c) COPYRIGHT Servicio de Publicaciones - Universidad de Murcia 